\title{
Balancing the book: Is it necessary and sufficient?
}

Authors: $\quad$ Prof Dudley Stark, Queen Mary University of London

Dr Dominic Cortis, University of Leicester/University of Malta

Contact Details: $\quad$ Dominic Cortis, Department of Mathematics, University of Leicester, University Road, Leicester, LE1 7RH, UK

t: +44 (0)116 2231794

e: dc156@le.ac.uk

Keywords: $\quad$ betting; solvency; odds; balancing the book; ruin 


\section{Abstract}

The solvency of bookmakers has only recently been placed in the spotlight even if it is a multibillion dollar sector. It is industry knowledge that bookmakers attempt to 'balance the book' by adjusting odds in relation to wagers made. This paper proves that the only way that profits are known with certainty is if and only if wagers are in proportion to probabilities implied by odds.

\section{Introduction}

The gross gambling yield, consisting of wagers less payouts, of sports betting was estimated to be around forty five billion euro in 2014 (H2 Gambling Capital, 2014). Any subcategory of the financial sector that may have amassed billions in transaction fees, the industry equivalent of gross gambling yield, would have raised significant interest with respect to its solvency regime. While solvency regimes have been created to regulate and harmonise international practices within the banking and insurance industries, the betting industry has not experienced a similar harmonization albeit the European Commission (n.d.) pointed out that "individual EU countries are unable to provide individuals with effective protection due to the nature of the online environment and the often cross-border dimension of online gambling". To our knowledge there is no current standard regulatory regime that formalizes a solvency capital requirement for bookmakers, even if they are not immune to risk.

Academic endeavors on betting markets have primarily focused on the client side, typically exploring why market participants themselves affect prices. Cortis (2015) changed the focus to the bookmaker in his paper proving the limitations set on odds through an evaluation of first and second moments. The first two findings were that the probabilities implied by odds offered on a market should be greater than actual probabilities and should add up to at least $100 \%$. Similar to Newall (2015), this work showed that bookmaker profits on accumulators are larger. Finally it was proved that bookmaker profit is guaranteed if wagers are kept in ratio with implied probabilities. In this paper, we extend the last finding to prove that the profits are known with certainty if and only if wagers are kept in ratio with implied probabilities.

'Balancing the book' is a term used to describe bookmakers' action of changing odds in relation to wagers. In layman terms, this paper validates such actions by proving that a bookmaker obtains a certain known profit only if wagers are in ratio with odds. This substantiates Kuypers' (2000) work that showed bookmakers may be better off moving from efficient to inefficient odds in order to capitalize on their clients' bias.

The next section of this paper describes how odds are set on an event. A knowledgeable reader would be aware of the terms used as such groundwork has been developed in greater detail in many other 
papers (e.g. Archontakis \& Osborne, 2007; Cortis, Hales \& Bezzina, 2013; Goddard \& Asimakopoulos, 2004). Notwithstanding, we think it is necessary to set the pace for a reader who may not be familiar with the betting industry. The following section proves our hypothesis and we conclude by giving a few examples.

\section{Setting odds}

Betting on outcomes can occur in a variety of set-ups ranging from a bet between friends to a high frequency exchange. However there are three main types of standard settings: an exchange, a parimutuel and a traditional bookmaker setting odds. In an exchange, market participants trade odds that they want to back (that is buy) or lay (sell) ${ }^{1}$. The exchange does not set odds but simply acts as a market and charges a percentage on winnings (Koning, 2009). On the other hand the winnings made on a bet placed in a pari-mutuel setting is in ratio of the wagers made on a particular outcome to the total wagers, less any commission by the organizer. For example a bettor who placed $€ 10$ on a horse, where a total of $€ 100$ was placed on this horse on an event with $€ 900$ wagered, would win $€ 90$ if the horse were to win the race. The organiser in both of these settings takes a part of the total wagers without having to calculate odds. However in the third traditional setting, a bookmaker sets odds and may have a risk of too many wagers placed a particular outcome such that the bookmaker suffers a loss if this occurs.

A bookmaker tries to maintain profit by offering odds that are lower than their true value. For example in a bet on tossing a fair die, a fair odd is at evens. However a bookmaker could offer the odd to win $€ 80$ for every $€ 100$ wagered. This means that the implied probability per outcome would be $\frac{100}{180}=55.56 \%$ and the sum of implied probabilities is greater than $100 \%$, being called the bookmaker margin (denoted $k)$. In this case, the bookmaker margin is $11.12 \%$.

In general, for an event assume there are $n$ mutually-exclusive possible outcomes, that is $[n]:=$ $\{1,2, \ldots, n\}$. Using the same notation of Cortis (2015), Shin (1993) and Štrumbelj (2014), we let $p_{i}$ denote the actual probability of outcome $i$ and let $\frac{1}{\pi_{i}}$ denote the odds offered on outcome $i$ as

\footnotetext{
${ }^{1}$ As an example consider a bettor (Bettor $A$ ) that wants to bet $f 100$ on England winning the world cup at odds of 10. Bettor A places this on the market (backs the outcome). On the other hand another bettor (bettor B) would be willing to be against England winning so places a lay (sell) order of $f 50$ at an odd of 9 . If these are the only trades placed on the market, then there is no agreement. However a third bettor might fancy a bet at an odd of 9 and hence backs $f 20$ at this odd. This is matched with Bettor's A earlier order. At this stage the market still has $f 30$ available to back at an odd of 9 and $£ 100$ to lay at an odd of 10 .
} 
determined in a European Odds format ${ }^{2}$. In the example above, the odd would be 1.8 and the implied probability $\left(\pi_{i}\right)$ is $55.56 \%$ for each outcome while the actual probability $\left(p_{i}\right)$ is $50 \%$. It was previously shown by Cortis (2015) that a bookmaker would risk a negative expected profit if the implied probability of any outcome was lower than the actual probability [Assumption 1]. This assumption implies that $\sum_{i=1}^{n} \pi_{i}>1$ an assumption in Cortis (2015). Ross (2011) states that if $\sum_{i=1}^{n} \pi_{i} \neq 1$, then arbitrage exists. However his example includes shortselling and assumes that a market is liquid. Cortis (2015) and our paper consider a scenario where bookmaker is setting odds which can be taken up by bettors. Hence assumption one applies. If the inverse was the case, that is individuals can 'lay' bets at odds offered by bettors, then $\sum_{i=1}^{n} \pi_{i}<1$ implies arbitrage exists. In a betting exchange, Ross' (2011) theorem holds. Moreover an odd should offer some form of profit to a bettor, that is $\frac{1}{\pi_{i}}>1$ [Assumption 2].

$$
\begin{array}{ll}
\text { ASSUMPTION 1: } & \pi_{i} \geq p_{i} \forall i \in[n] \\
\text { ASSUMPTION 2: } & \pi_{i}<1 \forall i \in[n]
\end{array}
$$

\section{Evaluating the variance in profit}

Suppose that $w_{i}$ units of money are bet on outcome $\mathrm{i}$. The total amount taken in by the bookmaker is $\sum_{i=1}^{n} w_{i}$ and the payout is $\frac{w_{i}}{\pi_{i}}$ if the outcome is $i$. Let $\mathrm{J}$ denote the outcome such that $\mathbb{P}(J=i)=p_{i}$ and let $R$ be the profit to the bookmaker. Hence $R=\sum_{i=1}^{n} w_{i}-\frac{w_{J}}{\pi_{J}}$. The probability distribution function of the profit is therefore

$$
\mathbb{P}(R=r)=\sum_{w_{i}=\pi_{i}\left(\sum_{i=1}^{n} w_{i}-r\right)}^{i n} p_{i}
$$

In setting odds the bookmaker has control over the implied probabilities $\left(\pi_{i}\right)$ but not the actual probabilities $\left(p_{i}\right)$ or wagers $\left(w_{i}\right)$. Hence we want to find for what possibilities of wagers are profits independent of the outcome that is known with certainty.

PROPOSITION. A bookmaker is guaranteed to make a profit of at least $\min _{i \in[n]}\left\{\sum_{i=1}^{n} w_{i}-\frac{w_{i}}{\pi_{i}}\right\} \cdot A$ bookmaker is guaranteed to make a profit if and only if the minimum is positive. That is:

$$
\mathbb{P}\left(R \geq \min _{i \in[n]}\left\{\sum_{i=1}^{n} w_{i}-\frac{w_{i}}{\pi_{i}}\right\}\right)=1 \text { and } \mathbb{P}(R>0) \text { if and only if } \min _{i \in[n]}\left\{\sum_{i=1}^{n} w_{i}-\frac{w_{i}}{\pi_{i}}\right\}>0
$$

\footnotetext{
${ }^{2}$ In European odds format, odds are shown as the inverse of probability.
} 
As an example uppose that $w_{i}$ are proportional to all $p_{i}$. Hence $w_{i}=K p_{i}$ for all $i \in[n]$. Then for all $i \in$ $[n]$,

$$
\begin{aligned}
& \sum_{i=1}^{n} w_{i}-\frac{w_{i}}{\pi_{i}}=K \sum_{i=1}^{n} p_{i}-K \frac{p_{i}}{\pi_{i}}=K\left(1-\frac{p_{i}}{\pi_{i}}\right) \text { and } \\
& \min _{i \in[n]}\left\{\sum_{i=1}^{n} w_{i}-\frac{w_{i}}{\pi_{i}}\right\}=K \min _{i \in[n]}\left(1-\frac{p_{i}}{\pi_{i}}\right)>0 \text { as Assumption (1) that states } \pi_{i} \geq p_{i} \forall i \in[n] .
\end{aligned}
$$

THEOREM. A bookmaker profit $R$ is independent of outcomes if and only if the wagers are proportional to the implied probabilities.

Proof: The amount of bookmaker profit is certain if the variability is zero.

$\operatorname{Var}(R)=\operatorname{Var}\left(\sum_{i=1}^{n} w_{i}-\frac{w_{J}}{\pi_{J}}\right)=\operatorname{Var}\left(\frac{w_{J}}{\pi_{J}}\right)$ since $\sum_{i=1}^{n} w_{i}$ is a constant.

Hence we need $\operatorname{Var}\left(\frac{w_{J}}{\pi_{J}}\right)=0$.

This is zero if and only if $\frac{w_{i}}{\pi_{i}}$ is a constant.

\section{Conclusion}

As an example consider an event with three mutually exclusive outcomes. Below we discuss a number of scenarios.

Scenario 1 [Odds offered of 5, 1.5 and 10]: This is not a possible combination as the combination of the three implied probabilities is lower than $100 \%$ (since $\sum_{i=1}^{n} \pi_{i}=\frac{1}{5}+\frac{1}{1.5}+\frac{1}{10}=96.67 \%$ ). This creates an opportunity for arbitrage such that a bettor that places 6,20 and 3 units on each respective outcome is bound to receive 30 units in winnings while having bet 29 , a certain profit.

Scenario 2 [Odds offered of 5, 0.8 and 10]: This is not a possible combination as anyone betting on the second outcome will receive less than their original stake (Assumption 2 fails).

Scenario 3[Odds offered 5, 1.2 and 10]: This is a possible combination and the bookmaker profit will depend on the wagers and the profits.

- If a unit bet is placed on each outcome, the bookmaker receives $\left(\sum_{i=1}^{n} w_{i}\right)$ three in wagers. They will pay off less than this if the second outcome occurs. 
- If wagers of 20,80 and 10 units are placed on each outcome, the bookmaker receives 110 units in wagers. The bookmaker would pay out 100 if the unlikely outcomes (odds of 5 and 10) occur but 96 if the likely one occurs. In every scenario the bookmaker will make a profit however it can fluctuate from 10 to 14 .

- Our theorem implies that only if wagers are placed in the ratio of $\frac{1}{5}: \frac{5}{6}: \frac{1}{10}$ would the profit be known with certainty. For example if 6, 25 and 3 units are wagered on each outcome respectively, the payout on any outcome would be 30 units and the profit is known to be 4 units.

In conclusion, our paper proves Cortis' (2015) earlier proposition and endorses the technique of 'balancing the book'. The limitation is that this finding relates mainly to single events and balancing the book on a series of accumulators/multiples, being bets placed on a series of events, would introduce more challenges due to the number of combinations at hand. 


\section{References}

Archontakis, F., \& Osborne, E. (2007). Playing it safe? A Fibonacci strategy for soccer betting. Journal of Sports Economics, 8(3), 295. Retrieved from http:// jse.sagepub.com/cgi/content/abstract/8/3/295

Cortis, D. (2015) Expected values and variances in bookmaker payouts: a theoretical approach towards setting limits on odds, Journal of Prediction Markets, 9(1), 1-14

Cortis, D., Hales, S., \& Bezzina, F. (2013). Profiting on inefficiencies in betting derivative markets: The case of UEFA EURO 2012. Journal of Gambling Business and Economics, 7(1), 39-51.

European Commission. (n.d.). Gambling. Website: http://ec.europa.eu/growth/sectors/gambling Accessed: 08 October 2016

Goddard, J., \& Asimakopoulos, I. (2004). Forecasting football results and the efficiency of fixed-odds betting. Journal of Forecasting, 23(1), 51-66. Retrieved from http:// dx.doi.org/10.1002/for.877 doi: $10.1002 /$ for. 877

H2 Gambling Capital. (2014, 11). Global gambling data and intelligence subscription service, global summary dataset.

Koning, R.H., \& van Velzen, B. (2009). Betting Exchanges: The Future of Sports Betting?. International Journal of Sport Finance 4(1), 42-62.

Kuypers, T. (2000). Information and efficiency: an empirical study of a fixed odds betting market Applied Economics 11(32), 1353-1363.

Newall, P. W. (2015). How bookies make your money. Judgment and Decision Making, 10(3), 225.

Ross, S. M. (2011). An Elementary Introduction to Mathematical Finance. Cambridge: Cambridge University Press.

Shin, H. S. (1993). Measuring the incidence of insider trading in a market for state contingent claims. Economic Journal, 103 (420), 1141-53.

Štrumbelj, E. (2014). On determining probability forecasts from betting odds. International journal of forecasting, 30(4), 934-943. 